\title{
USO DA CIF NA ÁREA DE AUDIOLOGIA: UMA REVISÃO INTEGRATIVA
}

\author{
INTERNATIONAL CLASSIFICATION OF FUNCTIONING, DISABILITY AND HEALTH IN
}

\author{
AUDIOLOGY
}

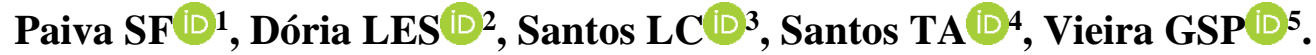

\begin{abstract}
${ }^{1}$ Doutoranda em Psicologia pela Universidade Federal de São João del-Rei (UFSJ). Docente na Universidade Federal de Sergipe (UFS), Lagarto, Sergipe, Brasil. E-mail: spaivafono@ academico.ufs.br. Lattes: http://lattes.cnpq.br/8004238855478961.

${ }^{2}$ Acadêmica em Fonoaudiologia na Universidade Federal de Sergipe (UFS), Lagarto, Sergipe, Brasil. E-mail: leedooria@gmail.com. Lattes: http://lattes.cnpq.br/3071899605813547.

${ }^{3}$ Acadêmica em Fonoaudiologia na Universidade Federal de Sergipe (UFS), Lagarto, Sergipe, Brasil. E-mail: laryssapixton@gmail.com. Lattes: http://lattes.cnpq.br/4558884134617583.

${ }^{4}$ Acadêmica em Fonoaudiologia na Universidade Federal de Sergipe (UFS), Lagarto, Sergipe, Brasil. E-mail: thaynarasantos152@yahoo.com. Lattes: http://lattes.cnpq.br/1663864126918614.

${ }^{5}$ Acadêmica em Fonoaudiologia na Universidade Federal de Sergipe (UFS), Lagarto, Sergipe, Brasil. E-mail: greicyvieira17@gmail.com. Lattes: http://lattes.cnpq.br/6559481025640021.
\end{abstract}

Correspondência: Scheila Farias de Paiva. Endereço: Av. Gov. Marcelo Déda, São José, Lagarto, Sergipe, Brasil, CEP: 49400-000. E-mail: spaivafono@academico.ufs.br.

\section{Recebido: $18 / 03 / 2021$}

Aceito: 29/04/2021

\section{RESUMO}

Introdução: A CIF é uma fermenta importante, que viabiliza a obtenção do perfil funcional, melhor escolha dos instrumentos de avaliação e a mensuração da incapacidade decorrente dos distúrbios auditivos e vestibulares. Objetivo: Apresentar estudos sobre a utilização da CIF no campo da Fonoaudiologia aplicada a área de audiologia. Metodologia: Revisão integrativa da literatura, realizada nas bases de dados eletrônicas BVS, Scielo, Lilacs e Medline, com tratamento qualitativo dos dados, descrevendo as características e relevância de cada estudo para descrição das informações coletadas. Resultados: Dos artigos válidos, cinco apresentam o uso da CIF na Audiologia em geral, dois artigos abordam a CIF no diagnóstico audiológico e na correlação da capacidade funcional com o desenvolvimento auditivo na reabilitação. Conclusão: Pode-se inferir que o uso da CIF revela-se viável na atuação do Fonoaudiólogo na área de audiologia e possibilita um olhar mais amplo para o tratamento tanto na área de audição quanto equilíbrio.

Palavras-chaves: Classificação Internacional de Funcionalidade, Incapacidade e Saúde. Fonoaudiologia. Audiologia.

\section{ABSTRACT}

Introduction: The ICF is an important instrument that makes possible to outline individuals' functioning profile, helps to better chose assessment instruments and provides a measurement of disabilities due to hearing and vestibular disorders. Objective: To present studies is use of ICF in the field of Speech Therapy applied to the audiology field. Methodology: Data was collected is electronic databases, such as BVS, Scielo, Lilacs and Medline, with qualitative treatment of the data. Results: From selected articles, total of 13, six of them were discarded due to duplicity; five, are related to the use of the ICF in Audiology; two, addressed this classification's description in Audiology; two, the audiological diagnosis, and one, the correlation between auditory functioning, auditory development and rehabilitation. Conclusion: ICF shows to be a great asset in the Audiology field, especially in the assessment and monitoring of auditory rehabilitation.

Keywords: International Classification of Functioning, Disability and Health. Speech Language and Hearing Sciences. Audiology. 


\section{INTRODUÇÃO}

A Classificação Internacional de Funcionalidade, Incapacidade e Saúde (CIF) é uma classificação de saúde e dos domínios relacionados à mesma, que geralmente é aplicada por profissionais da área da saúde e de outras áreas a fins, com a finalidade de referenciar sua prática profissional. O objetivo geral da CIF é favorecer uma linguagem unificada e padronizada como um sistema de descrição da saúde e de estados concernentes à saúde, viabilizando a comparação de dados relacionados a essas condições entre países, serviços, setores de atenção à saúde, assim como o rastreamento da sua evolução no tempo. ${ }^{1}$

Na Fonoaudiologia, a CIF contribui como norteadora das práticas referentes com os Distúrbios da Comunicação Humana. O uso da CIF em fonoaudiologia deve estar atrelado ao contexto clínico, já que os fatores biopsicossociais podem influenciar na relação de corpo e de qualidade de vida do indivíduo, o trabalho do fonoaudiólogo neste quesito é de agregar estes determinantes ambientais, sociais e econômicos ao serviço e ampliar a visão para todos os indivíduos abordados. ${ }^{2}$

Visto que a funcionalidade e a deficiência de uma pessoa ocorrem em uma conjuntura, a CIF também engloba uma relação de fatores ambientais. ${ }^{3}$ Os fatores contextuais são fatores ambientais e pessoais, foco central da classificação que se relaciona com seus impactos nas três dimensões das condições de saúde, sendo estas estrutura e função do corpo, atividade e participação social que compõem a primeira parte da CIF e para classificar a Funcionalidade e Incapacidade. A segunda parte, denominada Fatores Contextuais, é composta pelos Fatores Ambientais e Fatores Pessoais. Cada componente é especificado por um código alfanumérico identificados como 'b' para Funções do Corpo; 's' para Estruturas do Corpo; 'd' para Atividades e Participação e, 'e' para Fatores Ambientais. ${ }^{1,4}$

Embora a CIF seja um instrumento de classificação, os efeitos da perda auditiva e os resultados do tratamento, podem ser acompanhados por meio das categorias relacionadas aos níveis de comprometimento de funções e estruturas do corpo, atividade e participação. Dessa forma, a classificação pode auxiliar na organização e padronização das informações referentes ao perfil funcional e de qualidade de vida da pessoa com deficiência, especificamente a pessoa com deficiência auditiva. ${ }^{5}$

Na prática audiológica, a CIF viabiliza a obtenção do perfil de funcionalidade inicial do indivíduo, o acompanhamento da evolução dos mesmos, avaliação da abordagem terapêutica proposta e mensuração da incapacidade decorrente dos diferentes níveis de cada doença ou lesão, ou seja, permite o relacionamento das doenças à qualidade de vida do indivíduo. Quando estas adaptações são realizadas, busca-se que o indivíduo seja inserido na sociedade de forma que suas limitações não sejam o centro e sim o indivíduo na sua integralidade. O uso da CIF possibilita a coleta de dados e a utilização de critérios iguais, além da possibilidade de comparações internacionais. ${ }^{5}$

O objetivo desta pesquisa é verificar como ocorre o uso da CIF na prática clínica fonoaudiológica, em especial na área de audiologia apresentada na literatura científica entre o período de 2015 e 2021. 


\section{METODOLOGIA}

A pesquisa se constitui-se uma revisão integrativa da literatura sobre o uso da CIF na área de Fonoaudiologia, mais especificamente na subárea denominada Audiologia, tendo como pergunta de investigação: “O que a literatura traz sobre a utilização da Classificação Internacional de Funcionalidade, Incapacidade e Saúde na área da audiologia nos últimos anos?”.

A busca foi realizada em março de 2021 nas seguintes bases de dados: Scientific Eletronic Library Online (Scielo), Literatura Latino-Americana e do Caribe em Ciências da Saúde (LILACS), Medical Literature Analysis and Retrieval Sistem on-line (MEDLINE) e US National Library of Medicine National Institutes Health (PubMed).

Para a busca dos artigos foram utilizados os seguintes Descritores em Ciências da Saúde (DeCS/MeSH): “Classificação Internacional de Funcionalidade, Incapacidade e Saúde" e "audiologia", para a pesquisa também foi utilizado o operador boleano "AND”, com filtro para texto completo e para os idiomas português, inglês e espanhol.

Os critérios de inclusão foram: artigos disponíveis na íntegra, publicados em revistas científicas nas línguas português, inglês ou espanhol entre o ano de 2015 e março de 2021, com temática principal a relação da CIF com a audiologia, sem restrições para os anos de publicação. Os critérios de exclusão foram: artigos duplicados, pesquisas realizadas com a CIF em outas subáreas da fonoaudiologia.

A seleção dos artigos foi feita seguindo as seguintes etapas: pesquisa nas bases de dados com a estratégia de busca descrita anteriormente, seleção do material publicado de acordo com os critérios de inclusão e exclusão através da leitura de título e resumo; aplicação dos critérios de inclusão e exclusão na leitura completa do material selecionado e, por fim, análise e processamento do material por meio de um protocolo previamente elaborado para categorização dos estudos em ano e tipo de publicação, objetivo, características metodológicas do estudo, população de estudo e resultados. Por fim, tabulação e descrição das informações coletadas e realizada análise crítica dos dados, afim de ponderar as características e relevância de cada estudo.

\section{RESULTADOS}

De acordo com os critérios já mencionados anteriormente, foram encontrados 10 artigos conforme apresentado na FIGURA 1, e a descrição das características metodológicas de cada estudo conforme QUADRO 1, respectivamente. 


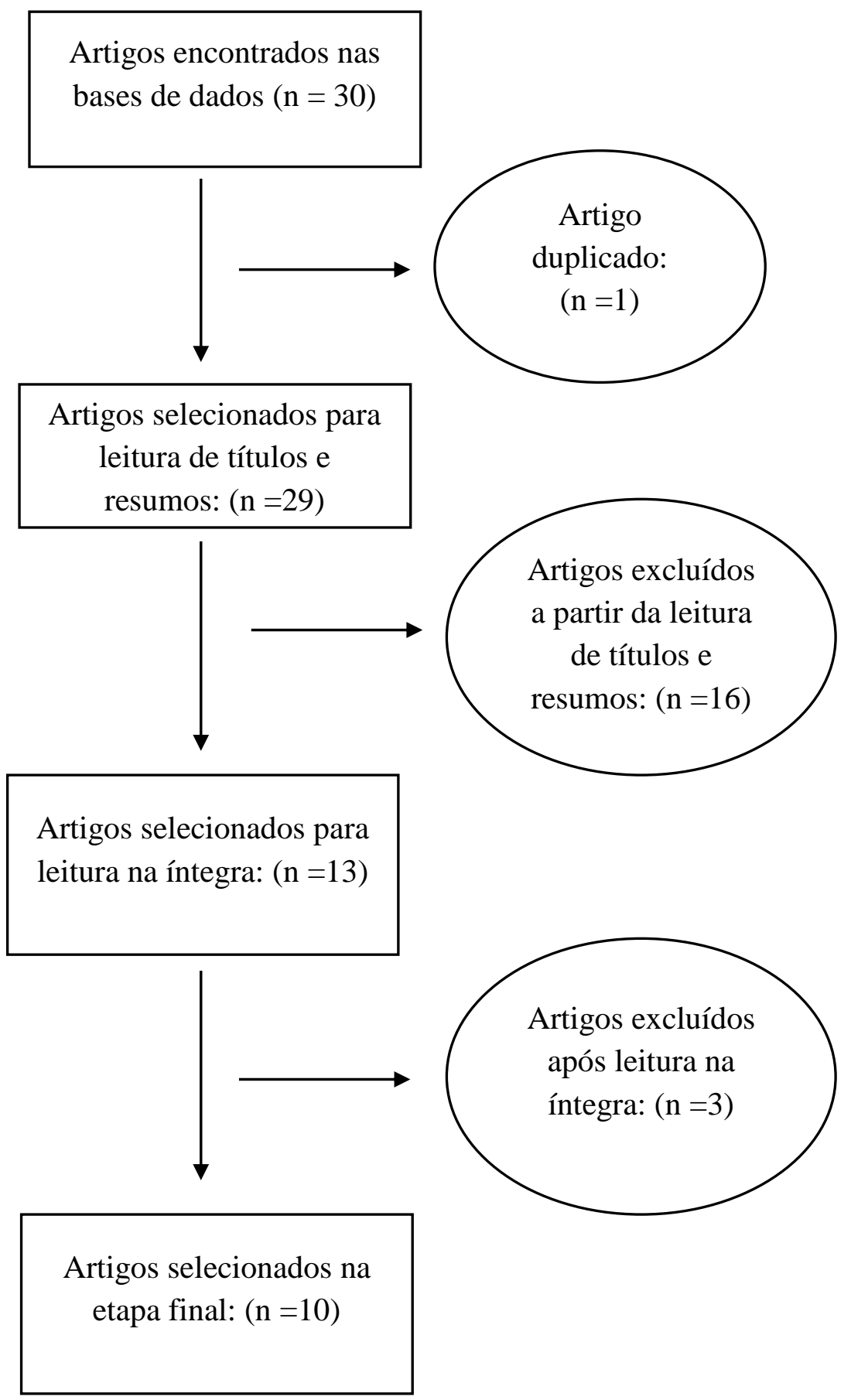

FIGURA 1 - Fluxograma de síntese da seleção de artigos para a revisão. 


\begin{tabular}{|c|c|c|c|c|c|}
\hline 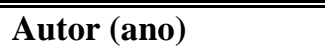 & Local & Natureza do estudo & $\bar{N}=$ participante & Idade dos participantes & "Contexto e Considerações \\
\hline $\begin{array}{l}\text { Bagatto MP, Moodie } \\
\text { ST }(2016)^{6}\end{array}$ & Canadá & Estudo de caso. & 1 & 2 anos. & $\begin{array}{l}\text { Descreve a relevância do uso da CIF-CJ em programas } \\
\text { de triagem auditiva, detecção e intervenção auditiva } \\
\text { precoce. }\end{array}$ \\
\hline $\begin{array}{l}\text { English K, Pajevic E } \\
(2016)^{7}\end{array}$ & $\begin{array}{l}\text { Estados } \\
\text { Unidos }\end{array}$ & Estudo de caso. & 1 & 13 anos & $\begin{array}{l}\text { Descreve como uma intervenção específica } \\
\text { (planejamento de transição para adolescentes) pode ser } \\
\text { documentada com a CIF-CJ. }\end{array}$ \\
\hline $\begin{array}{l}\text { Lind C, Meyer C, } \\
\text { Young J. }(2016)^{8}\end{array}$ & Austrália & Revisão Narrativa & Não se aplica & Não se aplica & $\begin{array}{l}\text { Descreve o uso da CIF e as implicações cotidianas de } \\
\text { deficiência intelectual e deficiência auditiva, com } \\
\text { particular respeito à avaliação da comunicação e opções } \\
\text { de intervenção. }\end{array}$ \\
\hline $\begin{array}{l}\text { Meyer C, Grenness C, } \\
\text { Scarinci N, Hickson } \\
\text { L. }(2016)^{9}\end{array}$ & Austrália & Estudo de casos & 2 & Sem dados & $\begin{array}{l}\text { Descreve a relevância e a aplicação da CIF à audiologia } \\
\text { juntamente com exemplos clínicos de aplicação na } \\
\text { avaliação e tratamento de crianças e adultos com perda } \\
\text { auditiva. }\end{array}$ \\
\hline $\begin{array}{l}\text { Grenness C, Meyer C, } \\
\text { Scarinci N, Ekberg K, } \\
\text { Hickson L. }(2016)^{10}\end{array}$ & Austrália & Estudos de casos & 1 & 82 anos & $\begin{array}{l}\text { Apresenta a aplicação da CIF na avaliação e o } \\
\text { tratamento audiológicos de um adulto idoso e compara } \\
\text { as abordagens com e sem a estrutura da CIF. }\end{array}$ \\
\hline $\begin{array}{l}\text { Bernardi SA, Pupo } \\
\text { AC, Trenche MCB, } \\
\text { Barzaghi L }(2017)^{11}\end{array}$ & Brasil & $\begin{array}{ll}\text { Codificação } & \text { de } \\
\text { instrumento } & \text { de } \\
\text { avaliação } & \end{array}$ & $\begin{array}{ll}13 \quad \text { (agentes } & \text { de } \\
\text { saúde) e } & 22 \\
\text { (crianças) } & \end{array}$ & $\begin{array}{l}\text { Entre } 1 \text { dia de vida aos } 11 \\
\text { meses. }\end{array}$ & $\begin{array}{l}\text { Correlaciona ítens do "Questionário para } \\
\text { monitoramento do desenvolvimento auditivo e de } \\
\text { linguagem no primeiro ano de vida"1 com os códigos } \\
\text { da CIF-CJ. }\end{array}$ \\
\hline
\end{tabular}

Continua...

${ }^{1}$ Alvarenga KF, Araújo ES, Melo TM, Martinez MAN, Bevilacqua MC. Questionário para monitoramento do desenvolvimento auditivo e de linguagem no primeiro ano de vida. CoDAS, 2013 [cited 2016 June 23];25(1):16-21. 


\begin{tabular}{|c|c|c|c|c|c|}
\hline $\begin{array}{l}\text { Van Leeuwen LM, } \\
\text { Pronk M, Merkus P, } \\
\text { Goverts ST, Anema } \\
\text { JR, Kramer SE. } \\
(2018)^{12}\end{array}$ & Holanda & $\begin{array}{l}\text { Desenvolvimento de } \\
\text { Cores sets }\end{array}$ & $\begin{array}{l}22 \text { (otorrinos e } \\
\text { audiologistas) e } 18 \\
\text { (indivíduos) } \\
\text { Total: da amostra: } \\
40\end{array}$ & $\begin{array}{l}\text { Variação da média } \text { de } \\
\text { idade: de } 31.6 \text { a } 52 \\
\text { (otorrinos } \\
\text { audiologistas) e } 54.5 \text { a } 58 \\
\text { (indivíduos). }\end{array}$ & $\begin{array}{l}\text { Desenvolve uma ferramenta baseada na CIF para Perda } \\
\text { Auditiva, operacionalizando suas categorias para } \\
\text { autorrelato do indivíduo. Identifica fatores facilitadores } \\
\text { e barreiras para o uso dessa ferramenta na percepção } \\
\text { dos profissionais de saúde auditiva e dos indivíduos. }\end{array}$ \\
\hline $\begin{array}{l}\text { Ranjan R, Bhat J, Vas } \\
\text { Naik PEM. }(2019)^{13}\end{array}$ & Índia & $\begin{array}{l}\text { Desenvolvimento de } \\
\text { instrumentos de } \\
\text { avaliação com base } \\
\text { na CIF }\end{array}$ & 36 participantes & 18 a 60 anos & $\begin{array}{l}\text { Descreve o desenvolvimento e validação de um } \\
\text { questionário com base na CIF, para avaliar a qualidade } \\
\text { de vida em indivíduos com vertigem ou tontura. }\end{array}$ \\
\hline $\begin{array}{l}\text { Van Leeuwen LM, } \\
\text { Pronk M, Merkus P, } \\
\text { Goverts ST, Terwee } \\
\text { CB, Kramer SE } \\
(2020)^{14}\end{array}$ & Holanda & $\begin{array}{l}\text { Validação de Core } \\
\text { Sets para deficiência } \\
\text { auditiva }\end{array}$ & 11 indivíduos & De 44 a 75 anos & $\begin{array}{l}\text { Apresenta um relato da implementação e validação de } \\
\text { uma ferramenta digital baseada na CIF, para avaliação } \\
\text { de adultos com problemas auditivos e auditivos. }\end{array}$ \\
\hline \begin{tabular}{lr}
\multicolumn{2}{l}{ Wittich W, Granberg } \\
S, Wahlqvist & M, \\
Pichora-Fuller & MK, \\
Mäki-Torkko & E \\
$(2021)^{15}$ &
\end{tabular} & $\begin{array}{l}\text { Canadá } \\
\end{array}$ & Revisão de escopo & Não se aplica & Não se aplica & $\begin{array}{l}\text { Apresenta e discute as variáveis que influenciam o uso } \\
\text { de tecnologia assistiva da perspectiva de pessoas com } \\
\text { surdocegueira, utilizando a estrutura da CIF para } \\
\text { contextualizar os achados. }\end{array}$ \\
\hline
\end{tabular}

QUADRO 1 - Análise dos artigos selecionados.

Fonte: Scielo, LILACS, MEDLINE PubMed (2021). 
Os resultados revelaram maior número de publicações em 2016 do que nos últimos quatro anos (FIGURA 2). Em relação aos países, a Austrália s destaca como o país com maior número de estudos neste sentido, contabilizando 3 (três) publicações em 2016; em segundo lugar o Canadá com 2 (dois) artigos, sendo 1 (um) em 2016 e outro em 2021, da mesma que a Holanda com 1 (um) artigo em 2018 e outro em 2020; em terceiro lugar os Estados Unidos em 2016, o Brasil com 1 (um) artigo em 2017 e a Índia no ano de 2019 respectivamente.

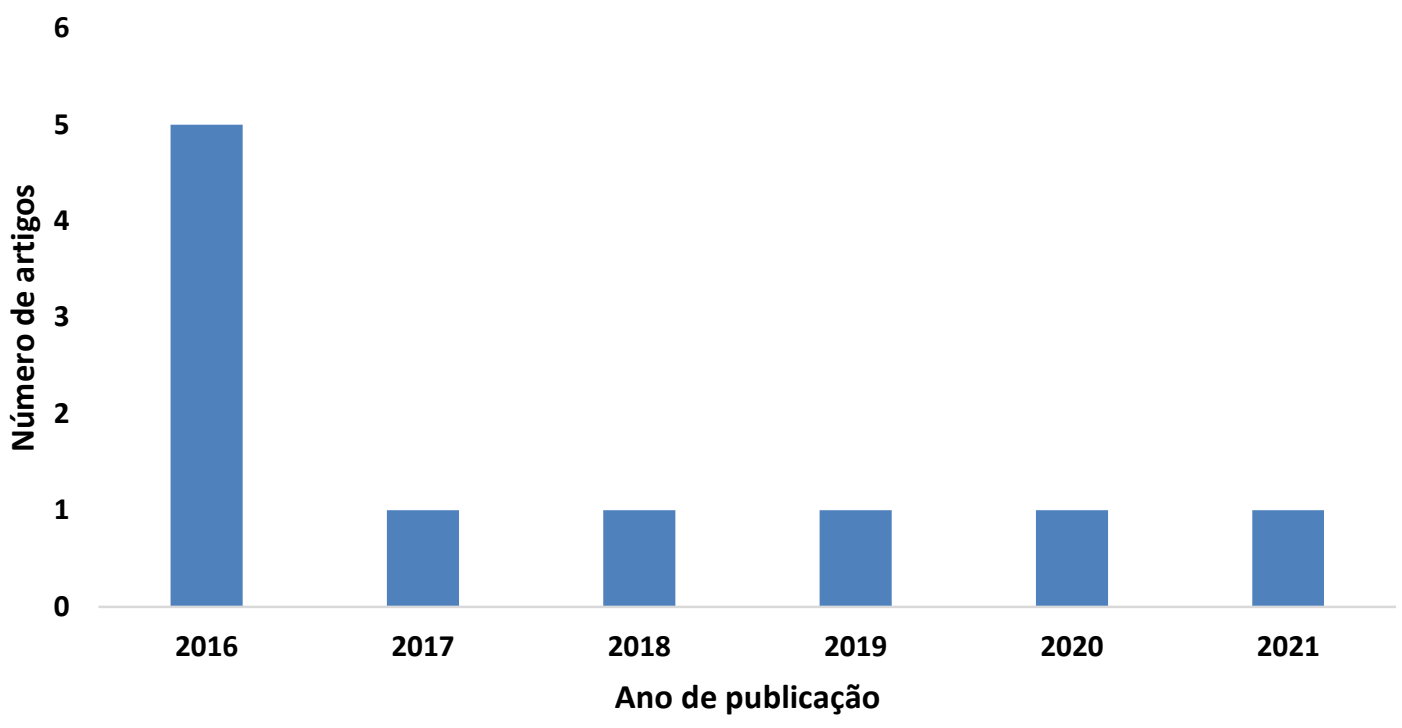

FIGURA 2 - Distribuição dos artigos sobre CIF na Audiologia do ano 2015-2021.

Em relação ao tipo de estudo a maioria encontrada é de natureza qualitativa distribuídas entre revisão de literatura sobre o tema, estudo de casos, desenvolvimento ou validação de Core Sets $^{2}$ e, codificação de itens ou ligação de instrumentos de avaliação ao conteúdo da CIF (FIGURA 3). O processo de construção de Core Sets tem sido muito utilizado para a obtenção de indicadores de funcionalidade em populações ou áreas de interesse específicas, desde que seguidas recomendações para da OMS para construção dos Core Sets.

De acordo com a análise dos dados, quanto ao tipo das publicações, percebe-se que a maior parte das publicações se divide em (a) adaptação de instrumentos de avaliação (4 artigos), (b) iniciativas apresentadas em forma de estudo de caso (4 artigos) e (c) a menor parte de revisão de literatura na área (2 artigos) .

\footnotetext{
${ }^{2}$ Conjuntos de categorias da CIF que descrevem a funcionalidade de pessoas com determinadas condições de saúde.
} 


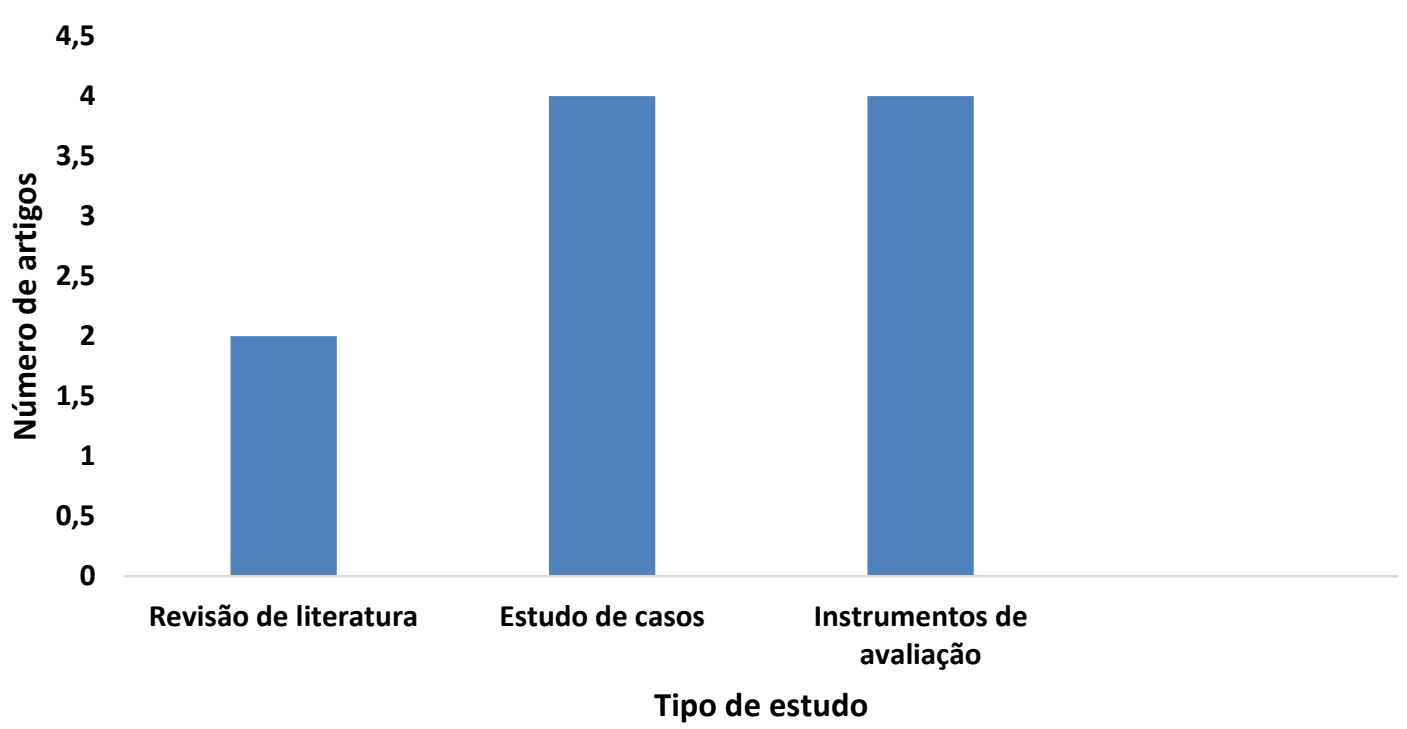

FIGURA 3 - Distribuição dos artigos por tipo de publicação (características metodológicas).

\section{DISCUSSÃO DOS DADOS}

Inicialmente, na análise dos dados fica evidente que Bernardi ${ }^{11}$ utiliza a Classificação Internacional de Funcionalidade, Incapacidade e Saúde para Crianças e Jovens (CIF-CJ) no serviço de triagem auditiva neonatal, para o registro e monitoramento do desenvolvimento da audição e da linguagem em crianças no primeiro ano de vida. Bagatto ${ }^{6}$ por sua vez, descreve a relevância da CIF-CJ para programas de triagem auditiva e oferece uma abordagem modificada, incluindo além dos aspectos auditivos, questões sobre a qualidade de vida e desenvolvimento humano ao longo do tempo, enquanto o de English ${ }^{7}$ descreve uma experiência de intervenção específica relacionada ao planejamento de transição para adolescentes documentada com a CIF-CJ.

Tendo em vista que a CIF-CJ é um instrumento que permite obter uma informação consistente e comparável internacionalmente sobre a experiência de saúde e de incapacidade, a colaboração destes estudos versam no sentido de subsidiar a abordagem da saúde preconizada pela OMS, exemplificado a viabilidade em relação a comparação de dados entre serviços, setores de atenção à saúde. Desta forma, apresenta-se como possibilidade, a CIF como ferramenta para o rastreamento e monitoramento da evolução de pacientes que pode ser acompanhada pelos profissionais em seus respectivos serviços de atenção à saúde auditiva ao longo de toda linha de cuidado, desde a idade mais tenra idade até o processo de transição para adolescência. $^{1,7}$

No estudo de Lind, ${ }^{8}$ o modelo da CIF foi aplicado para explorar as implicações cotidianas de pessoas com deficiência auditiva e deficiência cognitiva. Neste estudo a relevância e a aplicação da CIF à audiologia 
são descritas e sugeridas para mensuração do impacto psicossocial da deficiência auditiva em situações comunicativas cotidianas que não são mensuradas em sua maioria durante os testes audiológicos convencionais no momento da avaliação e tratamento de crianças e adultos com perda auditiva. Os autores destacam que a manifestação mais significativamente de uma perda ou redução da competência na comunicação falada cotidiana ocorrem em sua maioria nos componentes de atividade e participação e consequentemente, nas limitações de atividades autorrelatadas e restrições de participação do indivíduo em situações cotidianas.

Além dos autores supracitados, Grenness ${ }^{10}$ também realizou o estudo da CIF de forma comparativa e demonstrou como a CIF pode ampliar o olhar terapêutico para além da deficiência, principalmente ao se considerar as atividades, participação e fatores contextuais essenciais para a pessoa com a deficiência auditiva e sua família. Ao apresentar o contraste das duas versões de avaliação (uma versão sem a CIF como norteadora e outra com a CIF) para um mesmo caso, os autores demonstram como a CIF pode auxiliar na organização e padronização das informações referentes ao perfil funcional e de qualidade de vida da pessoa com deficiência auditiva, ao considerar todos estes aspectos da vida efetivamente. ${ }^{5}$

Por fim, os quatro últimos estudos abordam as barreiras e os facilitadores que colaboram para o uso ou abandono de dispositivos de acordo com as perspectivas das pessoas que possuem deficiência auditiva e visual. ${ }^{12-14,15}$ Os autores apresentaram como determinadas intervenções podem possibilitar o uso efetivo dos dispositivos, ${ }^{15}$ a necessidade de aprimoramento do atendimento com os indivíduos nas áreas de otologia e audiologia, ${ }^{12}$ como a operacionalização de uma ferramenta com base na CIF pode ser usada na prática de clínica em audiologia no que se refere à avaliação para adultos, ${ }^{14}$ bem como no desenvolvimento de um questionário para avaliação do impacto da tontura. ${ }^{13}$ Os facilitadores são parte fundamental na avaliação da funcionalidade de uma pessoa, pois a funcionalidade depende da relação do indivíduo com os fatores ambientais nos quais este encontra-se inserido ${ }^{3}$

Por fim, estudos de desenvolvimento de instrumentos ou validação de Core Sets e, codificação de itens ou ligação de instrumentos de avaliação ao conteúdo da $\mathrm{CIF}^{11-14}$ tem sido muito utilizado como forma de aproximação do conteúdo da CIF no processo da quebra de paradigmas ou transição efetiva para a prática clínica de acordo com o modelo biopsicossocial. Destaca-se, no entanto a necessidade de cautela nesta utilização e a leitura das recomendações para da OMS para construção de $\operatorname{Core~} \operatorname{Sets}^{16}$ a fim de permitir a integração de suas diversas dimensões (biológica, individual e social) proporcionando uma linguagem comum e coerente quanto à condição de saúde e dos estados a que essa se relaciona. ${ }^{17}$

\section{CONSIDERAÇÕES FINAIS}

Diante do exposto, apesar do número reduzido de artigos, percebe-se quanto ao uso da CIF na audiologia, a literatura nos revela possibilidades e viabilidade de aplicação desta ferramenta nos mais 
diversos contextos. Dentre os relatos estão contemplados serviços de triagem e monitoramento auditivo, acompanhamento do desenvolvimento auditivo e de linguagem através da capacitação de equipes e profissionais de saúde, assim como na adaptação de instrumentos de avaliação e monitoramento terapêutico para as diversas faixas-etárias. O uso da CIF na audiologia também promove efetivamente o cuidado centrado no indivíduo quando ao se considerar a influência dos fatores ambientais, a existência de facilitadores e o desempenho do indivíduo nas atividades e na participação social. Desta forma, de acordo com os artigos pesquisados, pode-se inferir que o uso da CIF, mesmo que de forma discreta, revela-se viável na atuação do fonoaudiólogo que atua na área de audiologia, bem como beneficia os indivíduos com deficiência auditiva que necessitem de uma abordagem mais ampla, com relação aos tratamentos na área de audição e equilíbrio.

\section{REFERÊNCIAS}

1. Organização Mundial da Saúde OMS. CIF: Classificação Internacional de Funcionalidade, Incapacidade e Saúde. São Paulo: Editora da Universidade de São Paulo - EDUSP; 2003.

2. Conselho Federal de Fonoaudiologia CFFa. Guia Norteador sobre a Classificação Internacional de Funcionalidade e Incapacidade/ CIF em Fonoaudiologia. Brasil; 2013.

3. World Health Organization WHO. The International Classification of Functioning, Disability and Health (ICF). Geneva, 2001.

4. Organização Mundial da Saúde Como usar a CIF: Um manual prático para o uso da Classificação Internacional de Funcionalidade, Incapacidade e Saúde (CIF). Versão preliminar para discussão. Genebra: OMS; 2013.

5. Morettin M, Bevilacqua M, Cardoso M. A aplicação da Classificação Internacional de Funcionalidade, Incapacidade e Saúde (CIF) na Audiologia. Distúrbios da Comunicação. 2008;20(3):395-402.

6. Bagatto MP, Moodie ST. Relevance of the International Classification of Functioning, Health and Disability: Children \& Youth Version in Early Hearing Detection and Intervention Programs. Semin Hear. 2016;37(3):257-71. DOI: https://doi.org/10.1055/s-0036-1584406.

7. English K, Pajevic E. Adolescents with Hearing Loss and the International Classification of Functioning, Health, and Disability: Children \& Youth Version. Semin Hear. 2016;37(3):247-56. DOI: https://doi.org/10.1055/s-0036-1584407.

8. Lind C, Meyer C, Young J. Hearing and Cognitive Impairment and the Role of the International Classification of Functioning, Disability and Health as a Rehabilitation Framework. Semin Hear. 2016;37(3):200-15. DOI: https://doi.org/10.1055/s-0036-1584410.

9. Meyer C, Grenness C, Scarinci N, Hickson L. What Is the International Classification of Functioning, Disability and Health and Why Is It Relevant to Audiology? Semin Hear. 2016;37(3):163-86. DOI: https://doi.org/10.1055/s-0036-1584412.

10. Grenness C, Meyer C, Scarinci N, Ekberg K, Hickson L. The International Classification of Functioning, Disability and Health as a Framework for Providing Patient- and Family-Centered Audiological Care for Older Adults and Their Significant Others. Semin Hear. 2016;37(3):187-99. DOI: https://doi.org/10.1055/s-0036-1584411.

11. Bernardi SA, Pupo AC, Trenche MCB, Barzaghi L. O uso da CIF no acompanhamento do desenvolvimento auditivo e de linguagem de crianças no primeiro ano de vida. Rev. CEFAC Online. 2017;19(2):159-170. DOI: http://dx.doi.org/10.1590/1982-021620171928016. 
12. Van Leeuwen LM, Pronk M, Merkus P, Goverts ST, Anema JR, Kramer SE. Barriers to and enablers of the implementation of an ICF-based intake tool in clinical otology and audiology practice. A qualitative pre-implementation study. PLoS ONE. 2018;13(12): e0208797. DOI: https://doi.org/10.1371/journal.pone.0208797.

13. Ranjan R, Bhat J, Vas Naik PEM. Quality of Life Rating for Dizziness: A Self-reporting Questionnaire. Indian J Otolaryngol Head Neck Surg. 2019 Nov;71(Suppl 2):1040-1046. DOI: https://doi.org/10.1007/s12070-017-1090-9.

14. Van Leeuwen LM, Pronk M, Merkus P, Goverts ST, Terwee CB, Kramer SE. Operationalization of the Brief ICF Core Set for Hearing Loss: An ICF-Based e-Intake Tool in Clinical Otology and Audiology Practice. Ear Hear. 2020;41(6):1533-1544. DOI: https://doi.org/10.1097/AUD.0000000000000867.

15. Wittich W, Granberg S, Wahlqvist M, Pichora-Fuller MK, Mäki-Torkko E. Device abandonment in deafblindness: a scoping review of the intersection of functionality and usability through the International Classification of Functioning, Disability and Health lens. BMJ Open. 2021;11(1):e044873. DOI: https://doi.org/10.1136/bmjopen-2020-044873.

16. Selb M, Escorpizo R, Kostanjsek N, Stucki G, Üstün B, Cieza A. A guide on how to develop an International Classification of Functioning, Disability and Health Core Set. Eur J Phys Rehabil Med. 2015;51(1):105-17. Epub 2014 Apr 1. PMID: 24686893.

17. Sociedade Brasileira de Fonoaudiologia. Respostas a perguntas frequentes sobre a Classificação Internacional de Funcionalidade, Incapacidade e Saúde (CIF): Saúde e a Fonoaudiologia. São Paulo, 2019. 